\title{
MORE ON SYMPLECTIC CORRECTORS
}

\author{
Robert I. MCLachlan
}

\begin{abstract}
.
We give a different point of view of the symplectic correctors of Wisdom, Holman, and Touma and study the condition that errors committed by the kernel can be corrected. Because of this requirement there are no outstandingly good new methods of this form, although any fourth-order kernel can be corrected for near-integrable problems. For systems that are not near-integrable we find that correctors bring no clear advantage.
\end{abstract}

\section{Symplectic correctors.}

Wisdom, Holman, and Touma [6] (hereafter WHT) have introduced and studied in depth the concept of a symplectic corrector, that is, a numerical integrator defined by an operator of the form

$$
e^{C} e^{K} e^{-C}
$$

in which the kernel $e^{K}$ is symplectic, and the corrector $e^{C}$ may or may not be. This is a useful form when many time steps can be taken without output, as then one only computes $e^{C}\left(e^{K}\right)^{n} e^{-C}$. Symplectic methods of this type were mentioned but not explored by Suzuki [5]; general methods of the form (1) were first introduced by Butcher [1] who found that for a fifth-order Runge-Kutta method, the kernel need only have five stages instead of the minimum of six needed for general RungeKuttas. However, the improvements are much more dramatic when the vector field is near-integrable and the kernel is a composition method, that is, the vector field to be integrated is of the form $A+B$ where $A$ and $B$ can be integrated exactly and $A=\mathcal{O}(1), B=\mathcal{O}(\epsilon)$. In this case WHT show that for all $n$ the dominant $\mathcal{O}\left(\epsilon t^{n}\right)$ errors can be eliminated by the corrector. WHT develop in depth one possible approach to the design of correctors and kernels for these problems; but they also comment that

We may also develop composition integrators which are intended from the beginning to be used with a corrector. Presumably, [this] is the most economical path to high order, since the kernel must satisfy a much reduced set of constraints, only that the error terms be correctable, not that they be zero.

1991 Mathematics Subject Classification. 65L05, 70-08.

Key words and phrases. Initial value problems, composition methods, operator splitting, symplectic integrators, Hamiltonian systems. 
In this note we explore this suggestion and also give a simple alternative derivation of symplectic correctors. WHT develop an "ideal" corrector and then approximate it by composition, the corrector to be used with various kernels; here we formulate and study the kernel and the corrector simultaneously. Regarding their hope expressed above, our conclusions are pessimistic: the "correctability constraint" does indeed restrict the choice of kernel, and seems to impose much the same order and stability limits as exist with ordinary composition methods. Our notation follows that of WHT, and in fact this is best read as a footnote to that paper. For most of the paper we have the near-integrable case in mind; the last section addresses the general case with regard to the above suggestion of WHT.

For the near-integrable case, the most important errors are those due to $\mathcal{L}_{A}^{m} B$ $(=[A,[A, \ldots,[A, B] \ldots]])$. These can be studied by considering the test problem $A=\partial_{x}, B=f(x) \partial_{y}$, for which the only nonzero Lie brackets, and hence errors, are due to $\mathcal{L}_{A}^{m} B$. A method which has order $n$ on this problem will have no errors due to $\mathcal{L}_{A}^{m} B, m<n$, when applied to arbitrary $A$ and $B$. For the test problem, a composition method of the form

$$
\ldots e^{b_{2} t B} e^{a_{2} t A} e^{b_{1} t B} e^{a_{1} t A}
$$

where $t$ is the time step, gives

$$
\begin{aligned}
& x \mapsto x^{\prime}=x+t \sum a_{i} \\
& y \mapsto y^{\prime}=y+t \sum b_{i} f\left(c_{i}\right)
\end{aligned}
$$

where $c_{i}=\sum_{j=1}^{i} a_{i}$; the true solution is

$$
\begin{aligned}
& x^{\prime}=x+t \\
& y^{\prime}=y+\int_{0}^{1} f(x+\alpha t) d \alpha
\end{aligned}
$$

So we have order $n$ if the $c_{i}$ are the nodes and the $b_{i}$ the weights of a quadrature formula of order $n$ on $[0,1]$. This may in turn be expressed by the quadrature conditions

$$
\sum b_{i} c_{i}^{m}=\frac{1}{m+1}, \quad m=0, \ldots, n-1
$$

obtained with the trial functions $f(x)=x^{m}$.

Now turn to corrector methods in which $e^{C}$ and $e^{K}$ both take the form (2). Let $k$ be the number of nodes (i.e., the number of applications of $e^{t B}$ in $e^{C}$ ). Fixing the kernel is the same as fixing some of the nodes and weights in the quadrature; if $e^{C}$ contributes a set of weights $\left\{b_{i}: i=1, \ldots, k\right\}$ and nodes $\left\{c_{i}\right\}$ then $e^{-C}$ contributes another set $\left\{-b_{i}\right\}$ and $\left\{c_{i}+1\right\}$, assuming the kernel is consistent. The necessity of using negative weights indicates that the correction process is rather ill-conditioned. A reasonable assumption is that the quadrature be symmetric about $x=\frac{1}{2}$; so $e^{C}$ must contain pairs $\pm b_{i}$ at nodes $\pm c_{i}$. The quadrature conditions for odd $m$ are then satisfied automatically. Figure 1(a) illustrates the symmetry of the nodes and weights.

\section{The leapfrog kernel.}


The corrector equations for any kernel can be written down immediately from (3). Take $e^{K}=e^{\frac{1}{2} t A} e^{t B} e^{\frac{1}{2} t A}$ which corresponds to a single node at $x=\frac{1}{2}$ with weight 1 , so the quadrature condition for $m=0$ is already satisfied. The contributions to (3) from the nodes at $\pm c_{i}$ cancel, leaving

$$
\left(\frac{1}{2}\right)^{m}+\sum_{i=1}^{k / 2} b_{i}\left(\left(1-c_{i}\right)^{m}-\left(1+c_{i}\right)^{m}\right)=\frac{1}{m+1}, \quad m=2,4,6, \ldots n-2
$$

If $n=k+2$ and the $c_{i}$ are given, distinct numbers, then these equations have a unique solution for the $b_{i}$. Furthermore, an exploration of the determining equations (4) suggests that no choice of nodes $c_{i}$ can increase the order $n$ beyond $k+2$, i.e., there is an order barrier for this type of quadrature. Since the efficiency of the corrector is not crucial we have not explored this point further.

The simplest, fourth-order corrector has $k=2$ and $c_{1} b_{1}=-\frac{1}{48}$. This quadrature is shown in Figure 1(a) for $c_{1}=\frac{1}{8}$. For higher orders, we follow WHT and take $c_{i}=i c, i=1, \ldots, k / 2$, obtaining a family of eighth-order correctors with $k=6$ :

$$
\begin{aligned}
& b_{1}=-\frac{155+3822 c^{2}+60480 c^{4}}{1935360 c^{5}} \\
& b_{2}=\frac{124+2352 c^{2}+12096 c^{4}}{1935360 c^{5}} \\
& b_{3}=-\frac{31+294 c^{2}+1344 c^{4}}{1935360 c^{5}}
\end{aligned}
$$

As $c$ decreases, $\max \left|b_{i}\right|$ increases $\left(b_{1} \approx-(0.15 / c)^{5}\right.$ as $\left.c \rightarrow 0\right)$, so some experimentation is needed to find the best value of $c$. The nodes $c_{i}$ may be taken in any order when reconstructing the $a_{i}$ in $(2)$; taking $\left(-c_{1}, c_{1},-c_{2}, c_{2} \ldots\right)$ gives the corrector

$$
\ldots e^{-c_{1} t A} e^{b_{1} t B} e^{2 c_{1} t A} e^{-b_{1} t B} e^{-c_{1} t A}
$$

as also used by WHT. The maximum step in $A$ is $2 i c$, so the high-order correctors of this family take large steps. Figure 1(b) illustrates the weights and placement of nodes for the sixteenth-order corrector with $k=14$ and $c=0.3$.

\section{Hybrid methods.}

In [4], methods are designed which eliminate the errors due to $\mathcal{L}_{A}^{m} B$ without using a corrector. One such class is based on Gaussian quadrature. Initial trials indicate that these are roughly as efficient as the corrector methods of the previous section, even in the limit of large numbers of steps between corrections. However, they also suggest a hybrid approach which may ameliorate some of the problems of the correctors used in WHT: use the accurate, stable methods of [4] to eliminate $\mathcal{L}_{A}^{m} B$ for small $m$, and leave the errors for larger $m$ to the corrector.

For example, take the kernel

$$
e^{\alpha t A} e^{\frac{1}{2} t B} e^{(1-2 \alpha) t A} e^{\frac{1}{2} t B} e^{\alpha t A}
$$


where $\alpha=(3-\sqrt{3}) / 6$. The first $\mathcal{O}(\epsilon)$ error is now at $\mathcal{O}\left(t^{5}\right)$, instead of $\mathcal{O}\left(t^{3}\right)$ for the leapfrog kernel. As before, one finds an eighth-order corrector with $k=6$ :

$$
\begin{aligned}
b_{1} & =\frac{40+819 c^{2}}{2177280 c^{5}} \\
b_{2} & =-\frac{32+504 c^{2}}{2177280 c^{5}} \\
b_{3} & =\frac{8+63 c^{2}}{2177280 c^{5}}
\end{aligned}
$$

These are much smaller in magnitude than with the leapfrog kernel; and for large $c$ they are $\mathcal{O}\left(c^{-3}\right)$ instead of $\mathcal{O}\left(c^{-1}\right)$. Figure 2 shows the largest weight for each kernel for a sixteenth-order corrector. This approach seems promising but a definite conclusion will depend on pinpointing the cause of the problems discussed in WHT.

\section{Higher orders.}

The corrector idea offers a possibility for avoiding some of the problems of highorder composition methods: that some $a_{i}$ and some $b_{i}$ must be negative for order more than 2 [2], and that the minimum number of stages needed rise rapidly with order - 3 applications of $e^{t B}$ are needed for fourth order, 7 for sixth order, and 15 for eighth order. To this end we studied fourth-order symplectic correctors.

We take a symmetric kernel to avoid destroying the symmetry of the quadrature:

$$
\begin{aligned}
e^{K} & =e^{\alpha_{r} t A} e^{\beta_{r} t B} \ldots e^{\alpha_{0} t A} \ldots e^{\beta_{r} t B} e^{\alpha_{r} t A} \\
\Rightarrow K & =t(A+B)+t^{3}\left(p_{1} \mathcal{L}_{A}^{2} B+p_{2} \mathcal{L}_{B}^{2} A\right)+\mathcal{O}\left(t^{5}\right)
\end{aligned}
$$

and the same symmetric corrector as before, with nodes $\pm a_{i}$ (the notation following WHT):

$$
\begin{aligned}
e^{X(a, b)} & =e^{a t A} e^{b t B} e^{-a t A} \\
\Rightarrow X(a, b) & =\exp \left(\mathcal{L}_{a t A}\right) b t B \\
e^{Z(a, b)} & =e^{X(a, b)} e^{X(-a,-b)} \\
\Rightarrow Z(a, b) & =2 \sinh \left(\mathcal{L}_{a t A}\right) b t B-t^{3} 2 a b^{2} L_{B}^{2} A+\mathcal{O}\left(t^{4}\right) ; \\
e^{C} & =\prod_{i=1}^{k / 2} e^{Z\left(a_{i}, b_{i}\right)} \\
\Rightarrow C & =2 t^{2}\left(\sum_{i=1}^{k / 2} a_{i} b_{i}\right) \mathcal{L}_{A} B-2 t^{3}\left(\sum_{i=1}^{k / 2} a_{i} b_{i}^{2}\right) \mathcal{L}_{B}^{2} A+\mathcal{O}\left(t^{4}\right) .
\end{aligned}
$$

For any kernel the errors corresponding to $t^{m+1} \mathcal{L}_{A}^{m} B$ can be eliminated as before, using the quadrature conditions (3). But now we must also eliminate all terms at order 3 and 4 -the order 4 terms are not trivial because the method (1) cannot itself be symmetric. We have

$$
e^{C} e^{K} e^{-C}=e^{W},
$$




$$
\begin{aligned}
W=K & +\mathcal{L}_{C} K+\mathcal{O}\left(t^{5}\right) \\
=t & (A+B)+t^{3}\left(p_{1} \mathcal{L}_{A}^{2} B+p_{2} \mathcal{L}_{B}^{2} A\right)+t^{3} 2\left(\sum_{i=1}^{k / 2} a_{i} b_{i}\right) \mathcal{L}_{A+B} \mathcal{L}_{B} A \\
& \quad+t^{4} 2\left(\sum_{i=1}^{k / 2} a_{i} b_{i}^{2}\right) \mathcal{L}_{A+B} \mathcal{L}_{B}^{2} A+\mathcal{O}\left(t^{5}\right)
\end{aligned}
$$

so, because the corrector cannot control the two third-order error terms independently, we obtain the third-order correctability condition on the kernel:

$$
p_{1}+p_{2}=0
$$

For example, if the kernel is itself third order then $p_{1}=p_{2}=0$ and correctability is ensured. More generally, an application of the Baker-Campbell-Hausdorff formula to $e^{K}$ gives

$$
4\left(p_{1}+p_{2}\right)=-\frac{1}{3}+\sum_{i=1}^{r}\left[\beta_{i}\left(\alpha_{0}+2 \sum_{j=1}^{i-1} \alpha_{j}\right)^{2}+\alpha_{i}\left(2 \sum_{j=1}^{i} \beta_{j}\right)^{2}\right]=0
$$

Although we have only one equation instead of the two required for a third-order kernel, it seems to impose much the same restrictions. With $r=1$ (for a two-stage kernel), we must have $\beta_{1}=\frac{1}{2}, \alpha_{1}=\left(1-\alpha_{0}\right) / 2$, and (7) simplifies to

$$
3 \alpha_{0}^{2}-3 \alpha_{0}+1=0
$$

which has no real solutions. For the simplest kernel, take $r=2$ and $\alpha_{2}=0$, $\alpha_{1}=\left(1-\alpha_{0}\right) / 2, \beta_{2}=\frac{1}{2}-\beta_{1}$ to get

$$
\alpha_{0}^{2} \beta_{1}-2 \alpha_{0} \beta_{1}^{2}+\beta_{1}\left(2 \beta_{1}-1\right)+\frac{1}{6}=0
$$

which has real solutions for $\beta_{1}<0$ and $\beta_{1} \geq \beta^{*}=1 /\left(2-2^{1 / 3}\right)$. The well-known three-stage fourth-order method corresponds to $\beta_{1}=\left(1-\beta^{*}\right) / 2$.

So including a corrector does allow new kernels, but there is no clear reason to use them over kernels which already have the required order [3]. One does get slightly shorter steps (e.g., the largest step when $\beta_{1}=-\frac{1}{2}$ is $\alpha_{0}=(\sqrt{93}-3) / 6 \approx 1.11$, compared to -1.70 for the fourth-order kernel), but this does not seem to balance the practical disadvantage of having to use a corrector. We conclude that the only application for symplectic correctors is the near-integrable case, for which extra, dominant errors can be eliminated by the corrector.

Having chosen a kernel one needs a corrector which leads to a method with overall fourth-order accuracy. From (6), the only new determining equation is $\sum_{i=1}^{k / 2} a_{i} b_{i}^{2}=0$. WHT solved this equation by taking $\pm a_{i}, \pm b_{i}$ pairs. This does not affect the rest of the determining equations; it merely corresponds to a "doubling up" of the quadrature nodes. Although it seems wasteful to use half the unknowns to satisfy one equation, an algebraic search for various values of $k$ did not uncover any better solutions. One piece of the corrector then takes the form

$$
e^{Z(a, b)} e^{Z(-a,-b)}=e^{a t A} e^{b t B} e^{-2 a t A} e^{-2 b t B} e^{2 a t A} e^{b t B} e^{-a t A}
$$


For example, three such pieces $(k=12)$ make an eighth-order corrector; taking the fourth-order kernel and $c_{i}=i / 3$ gives

$$
\begin{aligned}
& b_{1}=0.11365774925958663923, \\
& b_{2}=-0.07195437705518615839, \\
& b_{3}=0.01008366828359522585
\end{aligned}
$$

(the general, analytic solution is rather lengthy).

The above analysis can be extended to higher orders. For example, for a method with no $\mathcal{O}(\epsilon)$ or $\mathcal{O}\left(\epsilon^{2}\right)$ terms up to sixth order in $t$, one finds two correctability constraints on the three terms in question $\left(\mathcal{L}_{A}^{4} B, \mathcal{L}_{B} \mathcal{L}_{A}^{3} B\right.$, and $\left.\mathcal{L}_{A} \mathcal{L}_{B} \mathcal{L}_{A}^{2} B\right)$. Again they are satisfied if the three terms are already zero in the kernel.

\section{Extensions.}

Suppose the vector field cannot be split into two solvable parts. Then each of these methods corresponds naturally to a method of the same order involving an arbitrary first-order method and its inverse [3]. The only new case to be dealt with is the near-integrable one: when the vector field can be split as $A+B$ with $B=\mathcal{O}(\epsilon)$, but the vector field $B$ cannot be solved exactly. One might have to approximate its flow by, say, $\prod e^{t X_{i}}$ if $B=\sum X_{i}$. So suppose we have some approximation of the flow of $B$,

$$
e^{B^{+}}, \quad B^{+}=t B+t^{2} B_{2}+t^{3} B_{3}+\ldots,
$$

and its adjoint

$$
e^{B^{-}}, \quad B^{-}=t B-t^{2} B_{2}+t^{3} B_{3}-\ldots,
$$

where $B_{n}=\mathcal{O}\left(\epsilon^{n}\right)$.

If the method is second order, the only new lower-order error term is that due to $B_{2}$. This can be eliminated by replacing each $e^{t b B}, e^{t b B}$ pair in the method (1), (2) by the pair $e^{t b B^{+}}, e^{t b B^{-}}$. Any unpaired term (such as $e^{t B}$ in the leapfrog kernel) must be replaced by $e^{\frac{1}{2} t B^{+}} e^{\frac{1}{2} t B^{-}}$. Furthermore, such a replacement introduces no new $\mathcal{O}(\epsilon)$ terms at any order in $t$.

This approach is not sufficient at higher order. The $\mathcal{O}\left(\epsilon^{3} t^{3} B_{3}\right)$ term, for example, would be independent of the corrector, leading to a new constraint on the kernel. But there is a simple solution: just sequentially replace $e^{a t A} e^{b t B} e^{c t A} \ldots$ by $e^{a t A} e^{a t B^{+}} e^{(b-a) t B^{-}} e^{(b-a) t A} e^{(c-(b-a)) t A} \ldots$ in the kernel and corrector separately. For example, the piece of the corrector in Eq. (5) becomes

$$
\ldots \varphi\left(-c_{1} t\right) \varphi^{*}\left(\left(c_{1}+b_{1}\right) t\right) \varphi\left(\left(c_{1}-b_{1}\right) t\right) \varphi^{*}\left(-c_{1} t\right)
$$

where $\varphi(x)=e^{x A} e^{x B^{+}}$and the adjoint $\varphi^{*}(x)=e^{x B^{-}} e^{x A}$. Now from the general theory in [3], the overall order of the method is preserved; and the errors incurred in replacing $B$ by $B^{+}$or $B^{-}$are all $\mathcal{O}\left(\epsilon^{2}\right)$, so the new method still removes all $\mathcal{O}\left(\epsilon t^{n}\right)$ terms to the same order in $t$.

\section{Methods formed from symmetric stages.}

The existence of correctability constraints is most easily seen when the kernel and corrector are both composed of the same symmetric method - the standard 
approach for reaching high order [7]. Let $S(t)$ be symmetric, i.e., $S(t) S(-t)=1$, and take

$$
e^{K}=\prod_{i=1}^{r} S\left(w_{i} t\right), \quad e^{C}=\prod_{i=1}^{k} S\left(z_{i} t\right)
$$

Suppose

$$
S(t)=\exp \left(t X_{1}+t^{3} X_{3}+t^{5} X_{5}+\ldots\right) ;
$$

for the overall method there is one determining equation for each independent Lie bracket of the $X_{n}$. Take $e^{K}$ symmetric. The equations for the errors corresponding to the $X_{n}$ themselves are, for odd $n$,

$$
\delta_{n 1}=\sum_{i=1}^{r} w_{i}^{n}+\sum_{i=1}^{k} z_{i}^{n}+\sum_{i=1}^{k}\left(-z_{i}\right)^{n}=\sum_{i=1}^{r} w_{i}^{n}
$$

-i.e., the corrector makes no contribution. Thus these equations are included among the correctability conditions.

There are in fact no more at sixth order. To see this we have to actually construct a corrector. Solving the above equations provides a $K$ with

$$
K=t X_{1}+t^{5} k_{1} \mathcal{L}_{X}^{2} X_{3}+\mathcal{O}\left(t^{7}\right)
$$

where $k_{1}$ is given in [7]. To avoid even-order errors, one finds that $C$ must have no terms at order $t, t^{3}$, or $t^{5}$. Those due to $X_{1}, X_{3}$, and $X_{5}$ are avoided by taking $e^{C}=e^{D(t)} e^{D(-t)}$; that due to $\mathcal{L}_{X}^{2} X_{3}$ by taking $e^{D(t)}=e^{E(t)} e^{E(-t)}$ where now

$$
e^{E(t)}=\prod_{i=1}^{k / 4} S\left(z_{i} t\right)
$$

This gives

$$
C=t^{4} c_{1} \mathcal{L}_{X} X_{3}+\mathcal{O}\left(t^{6}\right), \quad c_{1}=4 \sum_{i=2}^{k / 4}\left[z_{i}^{3}\left(\sum_{j=1}^{i-1} z_{j}\right)-z_{i}\left(\sum_{j=1}^{i-1} z_{j}^{3}\right)\right] .
$$

Finally, the overall method has the expansion $K+\mathcal{L}_{C} K+\mathcal{O}\left(t^{9}\right)$, so the term $\mathcal{L}_{X}^{2} X_{3}$ can be corrected, if the corrector is chosen so that

$$
c_{1}=k_{1} \text {. }
$$

From the antisymmetry of $c_{1}$ we see that this equation has a solution for any $k_{1}$; for example, $k=8$ (leading to two unknowns $z_{1}, z_{2}$ ) is sufficient.

So the shortest sixth-order symplectic corrector has $r=5$ stages, as opposed to $r=7$ for uncorrected methods. The determining equations for the kernel have two real solutions (e.g., $w_{1} \approx 1.452, w_{2} \approx-2.151, w_{3} \approx 2.397$, with corrector $z_{1}=\frac{1}{4}$, $\left.z_{2} \approx-0.127\right)$ which are close to Yoshida's methods "B" and "C" [7]. Both of these are much less accurate than his method "A" - unfortunately this third method just fails to exist as a corrector. One can buy a little more freedom by going to $r=7$, e.g., $w_{1}=w_{2} \approx 0.431, w_{3} \approx-0.834, w_{4} \approx 0.944$ has slightly smaller coefficients that Yoshida's method A. Overall, it seems that dropping the determining equation $k_{1}=0$ from the kernel does not gain much. Extending the above derivation to $\mathcal{O}\left(t^{8}\right)$ 
shows that in addition to the $X_{7}$ term, $\mathcal{L}_{X_{3}}^{2} X_{1}$ cannot be corrected, so the kernel requires at least $r=9$ stages.

A corrector method essentially allows one to leave the secular part of the error to be dealt with later. One's intuition is that errors which can be made secular by the small change of coordinates corresponding to $e^{C}$ are not in general the most important ones. Our fairly thorough exploration of the determining equations at fourth and sixth order suggests that, indeed, the correctable portion of the error is neither large enough, nor a sufficiently significant constraint on the efficiency of the kernel, to make leaving it for later worth while.

Acknowledgements. I'd like to thank Jack Wisdom for his patient, extensive discussions of [6], and Jürgen Moser for inviting me to the Forschungsinstitut für Mathematik where this work was carried out.

\section{REFERENCES}

1. Butcher, J. C., The effective order of Runge-Kutta methods, Lecture Notes in Mathematics, vol. 109, Springer, 1969, pp. 133-139.

2. Goldman, D., and T. J. Kaper, Nth-order operator splitting schemes and nonreversible systems, preprint.

3. McLachlan, R. I., On the numerical integration of ordinary differential equations by symmetric composition methods, SIAM J. Sci. Comp. (1994) (to appear).

4. McLachlan, R. I., Composition methods in the presence of small parameters, BIT (submitted).

5. Suzuki, M., Improved Trotter-like formula, Phys. Lett. A 180 (1993), 232-234.

6. Wisdom, J., M. Holman, and J. Touma, Symplectic correctors, these proceedings.

7. Yoshida, H., Construction of higher order symplectic integrators, Phys. Lett. A 150 (1990), $262-268$

Figure 1. Placement of weights $b_{i}$ and nodes $c_{i}$ for two second-order methods. (a), $\mathcal{L}_{A}^{n} B$ errors removed for $n<4$ (the left two nodes form the corrector $e^{C}$ ); (b), for $n<16$. The bold line marks the quadrature domain $[0,1]$.

Figure 2. Largest weight $\left(=\max _{i}\left|b_{i}\right|\right)$ for two families of sixteenth-order correctors. The upper curve is for a leapfrog kernel, the lower curve for the two-stage kernel of section 3 .

Mathematics Department, Massey University, Palmerston North, New Zealand

E-mail address: rxm@boulder.colorado.edu 